\title{
Synthesis and styrene copolymerization of novel oxy ring-disubstituted isobutyl phenylcyanoacrylates
}

Megan Bailey, Margaret A. Barcinski, Adrian L. Galloway, Alyssa M. Guzman, Syed

Hussaini, Naimah A. Khatib, Elli D.R. Parisi, Tripp Tuff, Brockton D. Williams, Sara M.

Rocus, William S. Schjerven and Gregory B. Kharas

DePaul University, Chemistry and Biochemistry Department, 1110 West Belden Avenue, Chicago, IL 60614-3214

\begin{abstract}
Novel oxy ring-disubstituted isobutyl phenylcyanoacrylates, $\mathrm{RPhCH}=\mathrm{C}(\mathrm{CN}) \mathrm{CO}_{2} \mathrm{CH}_{2} \mathrm{CH}\left(\mathrm{CH}_{3}\right)_{2}$ (where $\mathrm{R}$ is 4-methoxy-2-methyl, 4-methoxy-3-methyl, 3-ethoxy-4-methoxy, 4-ethoxy-3-methoxy, 3,4-dibenzyloxy, 2-benzyloxy-3-methoxy, 3benzyloxy-4-methoxy, 2,3-methylenedioxy) were synthesized by the piperidine catalyzed Knoevenagel condensation of ring-disubstituted benzaldehydes and isobutyl cyanoacetate and characterized by $\mathrm{CHN}$ analysis, IR, ${ }^{1} \mathrm{H}$ and ${ }^{13} \mathrm{C}$ NMR. The acrylates were copolymerized with styrene in solution with radical initiation $(\mathrm{ABCN})$ at $70^{\circ} \mathrm{C}$. The compositions of the copolymers were calculated from nitrogen analysis and the structures were analyzed by FTIR, ${ }^{1} \mathrm{H}$ and ${ }^{13} \mathrm{C}$ NMR.
\end{abstract}


*Contact: gkharas@depaul.edu

\section{Introduction}

4-Methoxy-3-methyl ring-disubstituted ethyl phenylcyanoacrylate (PCA) is reported among the potent, orally bioavailable pyrimidine-5-carbonitrile-6-alkyl CXCR2 receptor antagonists [1] as well as in synthesis of methoxytolylsuccinic acids [2]. 3,4-

Diethoxyphenyl ethyl PCA is used in investigation of the kinetics and mechanism of oxidation of cyclic organic compounds in the liquid and solid phases [3]. 3,4Dimethoxyphenyl isoamyl PCA was a component of a photostable UV absorbent [4]. 3,4Diethoxyphenyl ethyl PCA was involved in synthesis of 3-hydroxypyridines [5]. 3Ethoxy-4-methoxyphenyl ethyl PCA was used in synthesis of 4-Benzyl-2imidazolidinones from $\mathrm{N}-[(1$-cyano-2-phenyl)ethyl] carbamates $[6,7]$ and 4-benzyl-2imidazolidinones [8]. 2-Benzyloxy-3-methoxy ethyl PCA was reported in preparation of (alkoxybenzyl)pyrrolidinone derivatives as nootropics [9]. 3-Methoxy-4-

(phenylmethoxy)phenyl ethyl PCA was involved in solvent-free Knoevenagel condensation under microwave irradiation in the presence of antimony trichloride [10, 11]. 3-Ethoxy-2-(phenylmethoxy) methyl PCA is used in preparation of methyl 2-oxo-2, 3-dihydrobenzo[b]oxepine-4-carboxylate [12]. 2,3-(Methylenedioxy) propyl PCA was involved in synthesis of 2-cyano-3-(3,4-methylene dioxyphenyl)-2-propionic acid ethyl ester [13]. This compound was used for synthesis of piperonal derivatives for intramolecular charge transfer material related to nonlinear optics [14, 15], as well as for a novel Golgi mannosidase inhibitor [16]. Earlier we have reported synthesis and styrene 
copolymerization a number of ring-disubstituted PCAs, such esters as methyl $[17,18]$, ethyl $[19,20]$, propyl [21], isopropyl [22, 23], butyl [24], isobutyl [25], methoxyethyl [26], and octyl [27].

Thus, in continuation of our investigation of novel PCA compounds we have prepared oxy ring-disubstituted isobutyl PCA, $\mathrm{RPhCH}=\mathrm{C}(\mathrm{CN}) \mathrm{CO}_{2} \mathrm{CH}_{2} \mathrm{CH}\left(\mathrm{CH}_{3}\right)_{2}$, where $\mathrm{R}$ is 4-methoxy2-methyl, 4-methoxy-3-methyl, 3-ethoxy-4-methoxy, 4-ethoxy-3-methoxy, 3,4dibenzyloxy, 2-benzyloxy-3-methoxy, 3-benzyloxy-4-methoxy, 2,3-methylenedioxy, and explored the feasibility of their copolymerization with styrene. To the best of our knowledge, there have been no reports on either synthesis of these compounds, nor their copolymerization with styrene [28].

\section{Experimental}

4-Methoxy-2-methyl, 4-methoxy-3-methyl, 3-ethoxy-4-methoxy, 4-ethoxy-3-methoxy, 3,4dibenzyloxy, 2-benzyloxy-3-methoxy, 3-benzyloxy-4-methoxy, 2,3methylenedioxybenzaldehydes, isobutyl cyanoacetate, piperidine, styrene, 1,1'azobis(cyclohexanecarbonitrile) (ABCN), and toluene supplied from Sigma-Aldrich Co., were used as received. Instrumentation is described in [29].

\section{Results and discussion}

\subsection{Synthesis and characterization of isobutyl phenylcyanoacrylates}

All isobutyl phenylcyanoacrylates (IPCA) compounds were synthesized by Knoevenagel condensation [30] of appropriate benzaldehydes with isobutyl cyanoacetate, catalyzed by base, piperidine (Scheme 1). 


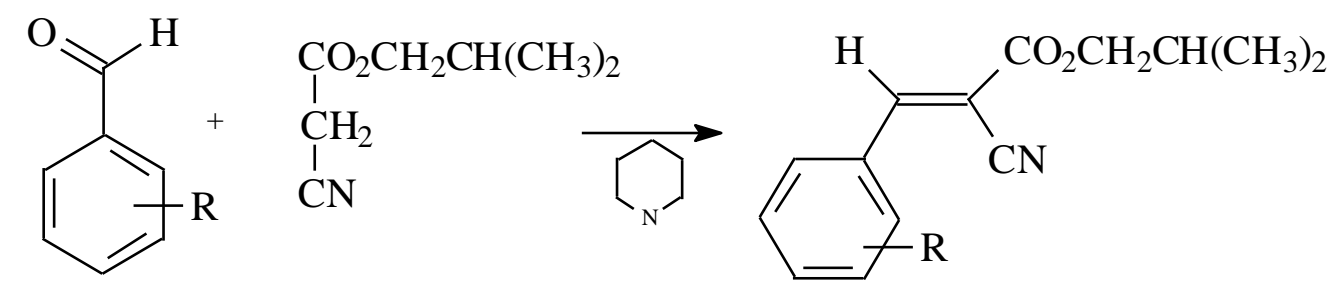

Scheme 1. Synthesis of isobutyl R-phenylcyanoacrylates, where R is 4-methoxy-2-methyl, 4-methoxy-3-methyl, 3-ethoxy-4-methoxy, 4-ethoxy-3-methoxy, 3,4-dibenzyloxy, 2benzyloxy-3-methoxy, 3-benzyloxy-4-methoxy, 2,3-methylenedioxy.

The preparation procedure was essentially the same for all the monomers. In a typical synthesis, equimolar amounts of isobutyl cyanoacetate and an appropriate benzaldehyde were mixed in equimolar ratio in a $20 \mathrm{~mL}$ vial. A few drops of piperidine were added with stirring. The reactions was allowed to proceed $48 \mathrm{hrs}$ at r.t. The product of the reaction was isolated by filtration and purified by crystallization from 2-propanol. The condensation reaction proceeded smoothly, yielding products, which were purified by conventional techniques. Melting points of the compounds in crystalline state were measured by DSC. The compounds were characterized by IR, ${ }^{1} \mathrm{H}$ and ${ }^{13} \mathrm{C}$ NMR spectroscopies. No stereochemical analysis of the novel ring-substituted IPCA was performed since no stereoisomers ( $E$ or/and $Z$ ) of known configuration were available.

\subsubsection{Isobutyl 4-methoxy-2-methylphenylcyanoacrylate}

Yield: $92.5 \%$; $\operatorname{mp} 79.1{ }^{\circ} \mathrm{C}$; ${ }^{1} \mathrm{H}$ NMR: $\delta 8.4(\mathrm{~s}, 1 \mathrm{H}, \mathrm{CH}=), 8.1,6.8,6.7(3 \mathrm{H}, \mathrm{Ph}), 4.1(\mathrm{~d}$, $\left.2 \mathrm{H}, \mathrm{CH}_{2}\right), 3.9\left(\mathrm{~s}, 3 \mathrm{H}, \mathrm{OCH}_{3}\right), 2.4\left(\mathrm{~s}, 3 \mathrm{H}, \mathrm{CH}_{3}\right), 2.3(\mathrm{~m}, 1 \mathrm{H}, \mathrm{CH}), 1.0\left(\mathrm{~d}, 6 \mathrm{H}, \mathrm{CH}_{3}\right) ;{ }^{13} \mathrm{C}$ 
NMR: $\delta 163(\mathrm{C}=\mathrm{O}), 152(\mathrm{HC}=), 142,134,132,122(\mathrm{Ph}), 115(\mathrm{CN}), 102(\mathrm{C}=), 72\left(\mathrm{CH}_{2}\right)$, $53\left(\mathrm{OCH}_{3}\right), 28(\mathrm{CH}), 20\left(\mathrm{PhCH}_{3}\right) 19\left(\mathrm{CH}_{3}\right)$; IR: $\left(\mathrm{cm}^{-1}\right)$ 3024-2819 (m, C-H), $2220(\mathrm{~m}$, $\mathrm{CN}$ ), 1713 (s, C=O), 1583 (s, C=C), 1289 (s, C-O-CH 3 ), 780, 760 (s, C-H out of plane). Anal. calcd. for $\mathrm{C}_{16} \mathrm{H}_{19} \mathrm{NO}_{3}$ : C, 70.31; H, 7.01; N, 5.12; Found: C, 69.06; H, 6.99; N, 5.36.

\subsubsection{Isobutyl 4-methoxy-3-methylphenylcyanoacrylate}

Yield $87 \%$; mp $111.0^{\circ} \mathrm{C} ;{ }^{1} \mathrm{H}$ NMR $\delta 8.2(\mathrm{~s}, 1 \mathrm{H}, \mathrm{CH}=), 7.9,7.8,6.9(3 \mathrm{H}, \mathrm{Ph}), 4.1(\mathrm{~d}, 2 \mathrm{H}$, $\left.\mathrm{CH}_{2}\right), 3.9\left(\mathrm{~s}, 3 \mathrm{H}, \mathrm{OCH}_{3}\right), 2.3\left(\mathrm{~s}, 6 \mathrm{H}, \mathrm{CH}_{3}\right), 2.0(\mathrm{~m}, 1 \mathrm{H}, \mathrm{CH}), 1.0\left(\mathrm{~d}, 6 \mathrm{H}, \mathrm{CH}_{3}\right) ;{ }^{13} \mathrm{C} \mathrm{NMR}$ $\delta 163(\mathrm{C}=\mathrm{O}), 153(\mathrm{HC}=), 134,133,128,124,112(\mathrm{Ph}), 117(\mathrm{CN}), 99(\mathrm{C}=), 72\left(\mathrm{CH}_{2}\right), 56$ $\left(\mathrm{OCH}_{3}\right), 27(\mathrm{CH}), 19\left(\mathrm{PhCH}_{3}\right), 17\left(\mathrm{CH}_{3}\right)_{2} ; \mathrm{IR}\left(\mathrm{cm}^{-1}\right): 3234-2812,2216(\mathrm{~m}, \mathrm{CN}), 1709$ (s, $\mathrm{C}=\mathrm{O}), 1665(\mathrm{C}=\mathrm{C}), 1228\left(\mathrm{~s}, \mathrm{C}-\mathrm{O}-\mathrm{CH}_{3}\right), 802,764$ (s, C-H out of plane). Anal. Calcd. for $\mathrm{C}_{16} \mathrm{H}_{19} \mathrm{NO}_{3}: \mathrm{C}, 70.31 ; \mathrm{H}, 7.01 ; \mathrm{N}, 5.12$; Found: C, 69.54; H, 6.82; N, 5.19.

\subsubsection{Isobutyl 3-ethoxy-4-methoxyphenylcyanoacrylate}

Yield 78\%; mp $83.0^{\circ} \mathrm{C} ;{ }^{1} \mathrm{H}$ NMR $\delta 8.2(\mathrm{~s}, 1 \mathrm{H}, \mathrm{CH}=), 7.8,7.5,6.9(\mathrm{~s}, 3 \mathrm{H}, \mathrm{Ph}), 4.2(\mathrm{q}, 2 \mathrm{H}$, $\left.\underline{\mathrm{C}}_{2} \mathrm{CH}_{3}\right), 4.1\left(\mathrm{~d}, 2 \mathrm{H}, \mathrm{CH}_{2}\right), 4.0\left(\mathrm{~s}, 3 \mathrm{H}, \mathrm{OCH}_{3}\right), 2.2(\mathrm{~m}, 1 \mathrm{H}, \mathrm{CH}), 1.3\left(\mathrm{t}, 3 \mathrm{H}, \mathrm{CH}_{3} \mathrm{CH}_{2}\right)$, $1.0\left(\mathrm{~d}, 6 \mathrm{H}, \mathrm{CH}_{3}\right) ;{ }^{13} \mathrm{C}$ NMR $\delta 161(\mathrm{C}=\mathrm{O}), 155(\mathrm{HC}=), 153,149,128,125,114,112(\mathrm{Ph})$, $115(\mathrm{CN}), 99(\mathrm{C}=), 72\left(\mathrm{CH}_{2}\right), 64\left(\underline{\mathrm{CH}}_{2} \mathrm{CH}_{3}\right), 56\left(\mathrm{OCH}_{3}\right), 28(\mathrm{CH}), 19\left(\mathrm{CH}_{3}\right), 14$ $\left(\mathrm{CH}_{2} \underline{\mathrm{CH}}_{3}\right)$; IR $\left(\mathrm{cm}^{-1}\right):$ 3000-2817 (m, C-H), 2216 (m, CN), 1713 (s, C=O), 1589 (C=C), 1275 (s, C-O-CH 3 ), 832, 752 (s, C-H out of plane). Anal. Calcd. for $\mathrm{C}_{17} \mathrm{H}_{21} \mathrm{NO}_{4}$ : C, 67.31; H, 6.98; N, 4.62; Found: C, 66.99; H, 6.66; N, 4.57. 


\subsubsection{Isobutyl 4-ethoxy-3-methoxyphenylcyanoacrylate}

Yield $82 \%$; mp $102.6^{\circ} \mathrm{C} ;{ }^{1} \mathrm{H}$ NMR $\delta 8.2(\mathrm{~s}, 1 \mathrm{H}, \mathrm{CH}=), 7.8,7.5,6.9(\mathrm{~s}, 3 \mathrm{H}, \mathrm{Ph}), 4.2(\mathrm{q}$, $\left.2 \mathrm{H}, \mathrm{C}_{2} \mathrm{CH}_{3}\right), 4.0\left(\mathrm{~d}, 2 \mathrm{H}, \mathrm{CH}_{2}\right), 3.9\left(\mathrm{~s}, 3 \mathrm{H}, \mathrm{OCH}_{3}\right), 2.1(\mathrm{~m}, 1 \mathrm{H}, \mathrm{CH}), 1.5(\mathrm{t}, 3 \mathrm{H}$, $\left.\mathrm{C}_{3} \mathrm{CH}_{2}\right), 1.0\left(\mathrm{~d}, 6 \mathrm{H}, \mathrm{CH}_{3}\right) ;{ }^{13} \mathrm{C} \mathrm{NMR} \delta 163(\mathrm{C}=\mathrm{O}), 155(\mathrm{HC}=), 153,149,128,124$, 112, $111(\mathrm{Ph}), 116(\mathrm{CN}), 99(\mathrm{C}=), 72\left(\mathrm{CH}_{2}\right), 65\left(\underline{\mathrm{CH}}_{2} \mathrm{CH}_{3}\right), 56\left(\mathrm{OCH}_{3}\right), 28(\mathrm{CH}), 20$ $\left(\mathrm{CH}_{3}\right), 15\left(\mathrm{CH}_{2} \mathrm{CH}_{3}\right) ; \mathrm{IR}\left(\mathrm{cm}^{-1}\right)$ : 3050-2818 (m, C-H), 2207 (m, CN), $1722(\mathrm{~s}, \mathrm{C}=\mathrm{O})$, 1624 (s, C=C), 1267 (s, C-O-CH 3 ), 820 (s, C-H out of plane). Anal. Calcd. for $\mathrm{C}_{17} \mathrm{H}_{21} \mathrm{NO}_{4}$ : C, 67.31; H, 6.98; N, 4.62; Found: C, 67.35; H, 7.06; N, 4.75.

\subsubsection{Isobutyl 3,4-dibenzyloxyphenylcyanoacrylate}

Yield 92\%; mp 91.1 ${ }^{\circ} \mathrm{C} ;{ }^{1} \mathrm{H}$ NMR $\delta 8.1(\mathrm{~s}, 1 \mathrm{H}, \mathrm{CH}=), 7.9-6.9(\mathrm{~m}, 3 \mathrm{H}, \mathrm{Ph}), 5.2(\mathrm{~s}, 4 \mathrm{H}$, $\left.\mathrm{PhCH}_{2}\right), 4.0\left(\mathrm{~s}, 2 \mathrm{H}, \mathrm{CH}_{2}\right), 2.2(\mathrm{~m}, 1 \mathrm{H}, \mathrm{CH}), 1.0\left(\mathrm{~d}, 6 \mathrm{H}, \mathrm{CH}_{3}\right) ;{ }^{13} \mathrm{C} \mathrm{NMR} \delta 163(\mathrm{C}=\mathrm{O})$, $155(\mathrm{HC}=), 136,128,127,125,113(\mathrm{Ph}), 115(\mathrm{CN}), 99(\mathrm{C}=), 72,71\left(\mathrm{CH}_{2}\right), 28(\mathrm{CH}), 20$ $\left(\mathrm{CH}_{3}\right)_{2}$; IR $\left(\mathrm{cm}^{-1}\right): 3100-2710(\mathrm{~m}, \mathrm{C}-\mathrm{H}), 2216(\mathrm{~m}, \mathrm{CN}), 1722(\mathrm{~s}, \mathrm{C}=\mathrm{O}), 1557$ (s, C=C), 1261 (s, C-O- $\mathrm{CH}_{3}$ ), 735, 698 (s, C-H out of plane). Anal. Calcd. for $\mathrm{C}_{28} \mathrm{H}_{27} \mathrm{NO}_{4}$ : C, 76.17; H, 6.16; N, 3.17; Found: C, 75.05; H, 6.10; N, 3.16.

\subsubsection{Isobutyl 2-benzyloxy-3-methoxyphenylcyanoacrylate}

Yield 83\%; mp 56.6 $6^{\circ} \mathrm{C},{ }^{1} \mathrm{H}$ NMR $\delta 8.5$ (s, 1H, CH=), 7.9-7.0 (m, 3H, Ph), 5.1 (s, 2H, $\left.\mathrm{PhCH}_{2}\right), 4.1\left(\mathrm{~d}, 2 \mathrm{H}, \mathrm{CH}_{2}\right), 3.9\left(\mathrm{~s}, 3 \mathrm{H}, \mathrm{OCH}_{3}\right), 2.0(\mathrm{~m}, 1 \mathrm{H}, \mathrm{CH}), 1.0\left(\mathrm{~d}, 6 \mathrm{H}, \mathrm{CH}_{3}\right) ;{ }^{13} \mathrm{C}$ NMR $\delta 162(\mathrm{C}=\mathrm{O}), 153(\mathrm{HC}=), 150,148,136,129,126,125,120,112(\mathrm{Ph}), 116(\mathrm{CN})$, $103(\mathrm{C}=), 72\left(\mathrm{PhCH}_{2}\right), 70\left(\mathrm{CH}_{2}\right), 55\left(\mathrm{OCH}_{3}\right), 29(\mathrm{CH}), 19\left(\mathrm{CH}_{3}\right)_{2} ; \mathrm{IR}\left(\mathrm{cm}^{-1}\right): 3008-2816$ (m, C-H), 2217 (m, CN), 1722 (s, C=O), 1605 (s, C=C), 1267 (s, C-O-CH3), 754, 698 (s, 
C-H out of plane). Anal. Calcd. for $\mathrm{C}_{22} \mathrm{H}_{23} \mathrm{NO}_{4}$ : C, 72.31; H, 6.34; N, 3.83; Found: C, 72.10; H, 6.40; N, 4.04.

\subsubsection{Isobutyl 3-benzyloxy-4-methoxyphenylcyanoacrylate}

Yield 79\%; mp $116.1^{\circ} \mathrm{C} ;{ }^{1} \mathrm{H}$ NMR $\delta 8.1(\mathrm{~s}, 1 \mathrm{H}, \mathrm{CH}=), 7.9-6.9(\mathrm{~m}, 3 \mathrm{H}, \mathrm{Ph}), 5.1(\mathrm{~s}, 2 \mathrm{H}$,

$\left.\mathrm{PhCH}_{2}\right), 4.0\left(\mathrm{~s}, 2 \mathrm{H}, \mathrm{CH}_{2}\right), 3.9\left(\mathrm{~s}, 3 \mathrm{H}, \mathrm{OCH}_{3}\right), 2.1(\mathrm{~m}, 1 \mathrm{H}, \mathrm{CH}), 1.0\left(\mathrm{~d}, 6 \mathrm{H},\left(\mathrm{CH}_{3}\right)_{2} ;{ }^{13} \mathrm{C}\right.$

NMR $\delta 163(\mathrm{C}=\mathrm{O}), 154(\mathrm{HC}=), 153,148,129,124,111(\mathrm{Ph}), 115(\mathrm{CN}), 104(\mathrm{C}=), 72,71$

$\left(\mathrm{CH}_{2}\right), 56\left(\mathrm{CH}_{3} \mathrm{O}\right), 28(\mathrm{CH}), 19\left(\mathrm{CH}_{3}\right)_{2} ; \mathrm{IR}\left(\mathrm{cm}^{-1}\right): 3112-2802(\mathrm{~m}, \mathrm{C}-\mathrm{H}), 2218(\mathrm{~m}, \mathrm{CN})$,

1720 (s, C=O), 1589 (s, C=C), 1263 (s, C-O-CH3), 945, 862 (s, C-H out of plane). Anal.

Calcd. for $\mathrm{C}_{22} \mathrm{H}_{23} \mathrm{NO}_{4}$ : C, 72.31; H, 6.34; N, 3.83; Found: C, 72.10; H, 6.40; N, 4.04.

\subsubsection{Isobutyl 2,3-methylenedioxyphenylcyanoacrylate}

Yield $76 \%$; mp $117.6{ }^{\circ} \mathrm{C} ;{ }^{1} \mathrm{H}$ NMR $\delta 8.4(\mathrm{~s}, 1 \mathrm{H}, \mathrm{CH}=), 7.8,7.0(3 \mathrm{H}, \mathrm{Ph}), 6.1(\mathrm{~s}, 2 \mathrm{H}$,

$\left.\mathrm{OCH}_{2} \mathrm{O}\right), 4.1\left(\mathrm{~d}, 2 \mathrm{H}, \mathrm{CH}_{2}\right), 2.1(\mathrm{~m}, 1 \mathrm{H}, \mathrm{CH}), 1.0\left(\mathrm{~d}, 6 \mathrm{H}, \mathrm{CH}_{3}\right) ;{ }^{13} \mathrm{C} \mathrm{NMR} \delta 162(\mathrm{C}=\mathrm{O})$, $154(\mathrm{HC}=), 153,149,147,122,120,114(\mathrm{Ph}), 115(\mathrm{CN}), 102(\mathrm{C}=), 101\left(\mathrm{OCH}_{2} \mathrm{O}\right), 73$

$\left(\mathrm{CH}_{2}\right), 28(\mathrm{CH}), 19\left(\mathrm{CH}_{3}\right)$; IR $\left(\mathrm{cm}^{-1}\right): 3002-2826(\mathrm{~m}, \mathrm{C}-\mathrm{H}), 2225(\mathrm{~m}, \mathrm{CN}), 1738$ (s, $\mathrm{C}=\mathrm{O}), 1610$ (s, C=C), 1242 (s, C-O-CH 3$), 782,678$ (s, C-H out of plane). Anal. Calcd. for $\mathrm{C}_{15} \mathrm{H}_{15} \mathrm{NO}_{4}: \mathrm{C}, 65.92 ; \mathrm{H}, 5.53 ; \mathrm{N}, 5.13$; Found: C, 65.74; H, 5.74; N, 5.75.

\subsection{Synthesis and characterization of styrene - IPCA copolymers}

Copolymers of the styrene (ST) and the IPCA compounds, P(ST-co-IPCA) were prepared in 25-mL glass screw cap vials at ST/IPCA $=3(\mathrm{~mol})$ the monomer feed using $0.12 \mathrm{~mol} / \mathrm{L}$ of $\mathrm{ABCN}$ at an overall monomer concentration $2.44 \mathrm{~mol} / \mathrm{L}$ in $10 \mathrm{~mL}$ of 
toluene. The copolymerization was conducted at $70^{\circ} \mathrm{C}$. After a predetermined time, the mixture was cooled to room temperature, and precipitated dropwise in methanol. The composition of the copolymers was determined based on the nitrogen content. The novel synthesized IPCA compounds copolymerized readily with ST under free-radical conditions (Scheme 2) forming white flaky precipitates when their solutions were poured into methanol. The conversion of the copolymers was kept between 10 and $20 \%$ to minimize compositional drift (Table 1). Nitrogen elemental analysis showed that between 17.8 and $30.0 \mathrm{~mol} \%$ of IPCA is present in the copolymers, which is indicative of relatively high reactivity of the IPCA monomers towards ST radical which is typical of oxy ring-substituted different esters PCA [16-20]. Since IPCA monomers do not homopolymerize, the most likely structure of the copolymers would be isolated IPCA monomer $(y=1)$ units alternating with short ST sequences $(x>1)($ Scheme 2).

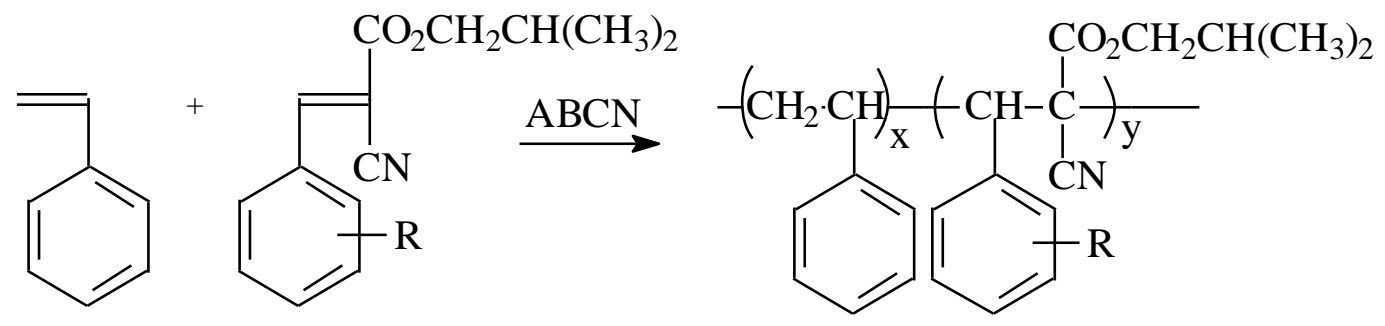

Scheme 2. Copolymerization of ST and the ring-substituted isobutyl phenylcyanoacrylates, $\mathrm{RPhCH}=\mathrm{C}(\mathrm{CN}) \mathrm{CO}_{2} \mathrm{CH}_{2} \mathrm{CH}\left(\mathrm{CH}_{3}\right)_{2}, \mathrm{R}=$ 4-methoxy-2-methyl, 4methoxy-3-methyl, 3-ethoxy-4-methoxy, 4-ethoxy-3-methoxy, 3,4-dibenzyloxy, 2benzyloxy-3-methoxy, 3-benzyloxy-4-methoxy, 2,3-methylenedioxy. 
Table 1. Copolymerization of isobutyl phenylcyanoacrylates with styrene.

\begin{tabular}{|c|c|c|c|c|c|c|c|c|c|}
\hline \multirow[b]{2}{*}{$\mathrm{R}$} & \multirow[b]{2}{*}{$\begin{array}{l}\text { Yield }^{\mathrm{a}} \\
\text { (wt\%) }\end{array}$} & \multirow[b]{2}{*}{$\underset{(\mathrm{wt} \%)}{\mathrm{N}}$} & \multirow[b]{2}{*}{$\begin{array}{l}\mathrm{m}_{2} \text { in } \\
\text { copol. } \\
\text { (mol\%) }\end{array}$} & \multirow[b]{2}{*}{$\begin{array}{l}\mathrm{M}_{\mathrm{w}} \\
(\mathrm{kD})\end{array}$} & \multirow[b]{2}{*}{$\begin{array}{c}\mathrm{T}_{\mathrm{g}} \\
\left({ }^{\circ} \mathrm{C}\right)\end{array}$} & \multicolumn{4}{|c|}{$T G A$} \\
\hline & & & & & & $\begin{array}{c}\text { Onset of } \\
\text { decomp. } \\
\left({ }^{\circ} \mathrm{C}\right)\end{array}$ & $\begin{array}{c}10 \\
\mathrm{wt} \% \\
\text { loss } \\
\left({ }^{\circ} \mathrm{C}\right)\end{array}$ & $\begin{array}{l}50 \\
\text { wt } \% \\
\text { loss } \\
\left({ }^{\circ} \mathrm{C}\right)\end{array}$ & $\begin{array}{l}\text { Residue } \\
\text { wt } \%\end{array}$ \\
\hline $\begin{array}{l}4-\mathrm{CH}_{3} \mathrm{O}, 2- \\
\mathrm{CH}_{3}\end{array}$ & 10.2 & 1.86 & 17.8 & 75.5 & 118 & 258 & 326 & 361 & 11.5 \\
\hline $\begin{array}{l}\text { 4- } \mathrm{CH}_{3} \mathrm{O}, 3- \\
\mathrm{CH}_{3}\end{array}$ & 13.3 & 2.07 & 20.5 & 76.0 & 129 & 256 & 318 & 353 & 4.6 \\
\hline $\begin{array}{l}3-\mathrm{C}_{2} \mathrm{H}_{5} \mathrm{O}, 4- \\
\mathrm{CH}_{3} \mathrm{O}\end{array}$ & 15.2 & 2.11 & 22.4 & $\begin{array}{l}79.9 \\
\end{array}$ & 129 & 278 & 323 & 356 & 4.0 \\
\hline $\begin{array}{l}4-\mathrm{C}_{2} \mathrm{H}_{5} \mathrm{O}, 3- \\
\mathrm{CH}_{3} \mathrm{O}\end{array}$ & 14.6 & 2.05 & 21.5 & 35.7 & 127 & 224 & 300 & 333 & 5.1 \\
\hline $\begin{array}{l}3,4- \\
\left(\mathrm{C}_{6} \mathrm{H}_{5} \mathrm{CH}_{2} \mathrm{O}\right)_{2}\end{array}$ & 15.6 & 2.08 & 31.0 & 49.8 & 83 & 242 & 308 & 353 & 30.8 \\
\hline $\begin{array}{l}2-\mathrm{C}_{6} \mathrm{H}_{5} \mathrm{CH}_{2} \mathrm{O}, \\
3-\mathrm{CH}_{3} \mathrm{O}\end{array}$ & 12.7 & 1.91 & 22.0 & 17.6 & 109 & 252 & 307 & 354 & 29.4 \\
\hline $\begin{array}{l}3-\mathrm{C}_{6} \mathrm{H}_{5} \mathrm{CH}_{2} \mathrm{O}, \\
4-\mathrm{CH}_{3} \mathrm{O}\end{array}$ & 16.2 & 1.95 & 22.8 & 40.7 & 110 & 241 & 316 & 358 & 24.25 \\
\hline $2,3-\mathrm{OCH}_{2} \mathrm{O}$ & 17.2 & 2.30 & 23.7 & 52.1 & 116 & 248 & 311 & 352 & 6.1 \\
\hline
\end{tabular}

The copolymers prepared in the present work are all soluble in ethyl acetate, THF, DMF and $\mathrm{CHCl}_{3}$ and insoluble in methanol, ethyl ether, and petroleum ether.

\section{Conclusions}

Novel oxy ring-substituted isobutyl phenylcyanoacrylates were prepared and copolymerized with styrene. The compositions of the copolymers were calculated from nitrogen analysis and the structures were analyzed by IR, $\mathrm{H}^{1}$ and ${ }^{13} \mathrm{C}$ NMR.

\section{Acknowledgments}

The authors are grateful to acknowledge that the project was partly supported by Chicago Society of Coating Technology (CSCT). 


\section{References}

1. The discovery of potent, orally bioavailable pyrimidine-5-carbonitrile-6-alkyl CXCR2 receptor antagonists. Porter, David W.; Bradley, Michelle; Brown, Zarin; Charlton, Steven J.; Cox, Brian; Hunt, Peter; Janus, Diana; Lewis, Sarah; Oakley, Paul; O'Connor, Des; et al. Bioorganic \& Medicinal Chemistry Letters (2014), 24(15), 32853290.

2. Methoxytolylsuccinic acids. Vyas, V. A.; Bokil, K. V.; Nargund, K. S. Journal of the University of Bombay, Science: Physical Sciences, Mathematics, Biological Sciences and Medicine (1940), 9(Pt. 3), 140-4.

3. Investigation of the kinetics and mechanism of oxidation of cyclic organic compounds in the liquid and solid phases. I. Investigation of the oxidizability of organic compounds under isothermal conditions. Tkacheva, A. P.; Karitonov, G. V. Izvest. Akad. Nauk Kirgiz. S.S.R., Ser. Estestven. i Tekh. Nauk (1960), 2(No. 5), 71-83.

4. Photostable UV absorbent containing $\alpha$-cyanocinnamyl moiety. Wang, Chenjie; Chaudhuri, Ratan K.; Jachowicz, Janusz; Locke, Bruce C.; Miksza, Frank M. U.S. (1998), US 5830441 A 19981103.

5. Synthesis of 3-hydroxypyridines. I. Condensation of aromatic aldehydes with ethyl cyanoacetate. Popp, Frank D. Journal of Organic Chemistry (1960), 25, 646-7.

6. 4-Benzyl-2-imidazolidinones from N-[(1-cyano-2-phenyl)ethyl] carbamates. Gruenman, Vsevolod; Hoffer, Max. U.S. (1975), US 3923833 A 19751202.

7. Substituted benzylethylenedicarbamates. Gruenman, Vsevolod; Hoffer, Max. U.S. (1975), US 3892777 A 19750701. 
8. 4-Benzyl-2-imidazolidinones. Gruenman, Vsevolod; Hoffer, Max. Ger. Offen. (1971), DE 2108438 A 19710909.

9. Preparation of (alkoxybenzyl)pyrrolidinone derivatives as nootropics. Goto, Giichi; Nagaoka, Akinobu. Jpn. Kokai Tokkyo Koho (1987), JP 62116557 A 19870528.

10. Solvent-free Knoevenagel condensation reaction under microwave irradiation exploiting a new reagent: antimony trichloride. Mitra, Alok Kumar; Karchaudhuri, Nilay; De, Aparna. Journal of the Indian Chemical Society (2005), 82(2), 177-179.

11. Solvent-free microwave enhanced Knoevenagel condensation of ethyl cyanoacetate with aldehydes. Mitra, Alok Kumar; De, Aparna; Karchaudhuri, Nilay. Synthetic Communications (1999), 29(16), 2731-2739.

12. Application of the acetate of Baylis-Hillman adducts of salicylaldehydes in the synthesis of methyl 2-oxo-2,3-dihydrobenzo[b]oxepine-4-carboxylates. Ahn, Sang-Hyun; Lim, Hee Nam; Lee, Kee-Jung. Journal of Heterocyclic Chemistry (2008), 45(6), 17011706.

13. New organic crystal material for SHG, 2-cyano-3-(3,4-methylene dioxy phenyl)-2propionic acid ethyl ester(CMPE). Mori, Yasushi; Sano, Kenji; Todori, Kenji; Kawamonzen, Yosiaki. Proceedings of SPIE-The International Society for Optical Engineering (1991), 1560(Nonlinear Opt. Prop. Org. Mater. 4), 310-14.

14. Organic optical nonlinear material. Mori, Yasushi; Sugiuchi, Masami. Jpn. Kokai Tokkyo Koho (1988), JP 63163827 A 19880707. 
15. A new intramolecular charge transfer material for nonlinear optics: piperonal derivatives. Mori, Y.; Okamoto, M.; Wada, T.; Sasabe, H. Materials Research Society Symposium Proceedings (1988), 109(Nonlinear Opt. Prop. Polym.), 345-50.

16. A novel Golgi mannosidase inhibitor: Molecular design, synthesis, enzyme inhibition, and inhibition of spheroid formation. Koyama, Ryosuke; Kano, Yui; Kikushima, Kaori; Mizutani, Ayaka; Soeda, Yuta; Miura, Kazuki; Hirano, Takako; Nishio, Toshiyuki; Hakamata, Wataru. Bioorganic \& Medicinal Chemistry (2020), 28(11), 115492.

17. Novel Copolymers of Styrene. 14. Some Ring-Disubstituted Methyl 2-Cyano-3-Phenyl2-Propenoates. G.B. Kharas, A. Shahbein, J.L. Fabbri, C.E. Fanter, D.R. Garcia, M.G. Ibarra, D. Lane, E. Marshall, E.E. Pierce, I.F. Sanchez, B.C. Sandoval, and A.D. Santi. J. Macromol. Sci. A51(5) 394-398 (2014).

18. Novel Copolymers of Styrene and Dialkoxy Ring-substituted Methyl 2-cyano-3-phenyl2-propenoates. G.B. Kharas, J.L. Christensen, D.J. Cichanski, K.E. Goldman, C.L. Gordon, L.M. Knowles, C.N. Krefft, M. Matouk, and K. Watson. J. Macromol. Sci., 43 (7) 989-994 (2006).

19. Novel Copolymers of Styrene. 6. Alkoxy Ring-substituted Ethyl 2-Cyano-3-phenyl-2propenoates.G.B. Kharas, A.A. Delgado, N.E. Anderson, A. Bajor, A.C. Colbert, A. Coleman, C.E. Gregory, J. Hayes, J. Lantin, J.M. Malecki, T.C. Murphy, A. Oprescu, B.F. Rydzon, and I. Timoshevskaya. J.Macromol. Sci. A50 (3) 276-280 (2013).

20. Novel Copolymers of Styrene. 2. Some Ring-substituted Ethyl 2-Cyano-3-phenyl-2propenoates. G. B. Kharas, E.S. Molina, B. Bobot, J. Bueno, J. Carney, J.Y.C. Chung, 
K.N. Krerowicz, T. Miller, S.L.I. Mills, M.A. Stankovich, I. Syed, C.S. Vaidya, and P. Wesolowski. J. Macromol. Sci. A50 (1) 1-5 (2013).

21. Novel Copolymers of Styrene. 6. Some Ring-Disubstituted Propyl 2-Cyano-3-Phenyl-2-

Propenoates. G.B. Kharas, L.A. Alyahya, S. M. Rocus, A. Ismail, R. Juarez, A. Kavaliauskaite, K.Y. Lechuga, A.C. Leeper, and G.C. Lenti. J. Macromol. Sci. A53(12) 725-728 (2016).

22. Synthesis and styrene copolymerization of novel oxy ring-disubstituted isopropyl phenylcyanoacrylates. J.L. Zepeda, M.M. Betts, S. Dalloul, A.G. Gonzalez, J.K. Henning, R.X. Liu, J.J. Ludtke, J.R. Mihai, S.M. Rocus, W.S. Schjerven, and G.B. Kharas. J. Phys. Chem. Res. 1(2) 1-5 (2019).

23. Synthesis and styrene copolymerization of novel ring-disubstituted isopropyl cyanoacrylates. P.M. Whelpley, S. Bajramovic, D.M. Bracamontes, G.A. Buechner, A.D. Eremin, E.J. Kowalczyk, D.D. Lender, R. McCann, W.S. Schjerven, G.B. Kharas. ChemRxiv (2019), 1-15. https://doi.org/10.26434/chemrxiv.9891161.v1.

24. Novel Copolymers of Styrene. 5. Methyl and Methoxy Ring-Disubstituted Butyl 2Cyano-3-Phenyl-2-Propenoates. G.B. Kharas, H. Feng, L.A. Arendt, S.E. Belton, M.Q. Edwards, E.K. Franz, A. Grin, C.M. Hale, I.C. Oyeyipo, A.L. Wolske, P.L. Zavala, and A. Zenunovic. J. Macromol. Sci. A52 (12) 976-981 (2015).

25. Synthesis and styrene copolymerization of novel ring-substituted isobutyl phenylcyanoacrylates. Abuelroos, Samer A., Francis, Divya V., Fricano, Adriana M., Gaona, Mariana, Huene, Jason T., Nagani, Jawairia A., Pecoraro, Cristina M., Rivero, Dayessi L., Stone, Sydney A., Udine, Marissa R., Rocus, Sara M., Schjerven, William 
S., and Kharas, Gregory. ChemRxiv Preprint (11.11.2020).

https://doi.org/10.26434/chemrxiv.11401347.v4

26. Synthesis and styrene copolymerization of novel trisubstituted ethylenes: 7. Some ringdisubstituted 2-methoxyethyl phenylcyanoacrylates. Imaad Allahrakha, Vikram Bhagavat, Justin Bodner, Deanna M. Briones, Jacqueline C. Calderon, Nadia Quad, Abdul Rafay, Claire M. Sagartz, Sid Sarfaraz, Adam W.T. Steffeck, Sabah M.

Sulaiman, Stephanie L. Taiberg, Skyler M. Thompson, Joanna L. Torres, Sara M.

Rocus, William S. Schjerven, Gregory B. Kharas. ChemRxiv. Preprint. (26.01.2021) https://doi.org/10.26434/chemrxiv.13262660.v7

27. Synthesis and styrene copolymerization of novel trisubstituted ethylenes: 6. Methyl, halogen and oxy ring-disubstituted octyl phenylcyanoacrylates. Emma J. Clajus, Natalie Cote, Rama Dalloul, Giulia M. DiMarco, Mollie J. Eriksson, Yesenia Garcia, Nathalie A. Gijsbers, Jay H. Kaila, Amina S. Malik, Madeeha I. Mohiuddin, Anaa Mulk, Neil T. Patel, Sara M. Rocus, William S. Schjerven, and Gregory B. Kharas. ChemRxiv. Cambridge Open Engage Version 17 Jul 11, 2021. https://doi.org/10.33774/chemrxiv-2021-k812v-v17

28. SciFinder Structure Search Feb 18, 2022.

29. Synthesis and styrene copolymerization of novel alkyl ring-substituted isobutyl 2-cyano3-phenyl-2-propenoates. Schjerven, William S.; Groy, Randi; Antonishina, Yekaterina S.; Black, Claire I.; Bolin, Madysen P.; Caniglia, John; Daroach, Deepak P.; Farooqui, Farah T.; Feiz, Yalda; Garcia, Sam; Gasparini, Gabriella; Kharas, Gregory B. Abstracts 
of Papers, 259th ACS National Meeting \& Exposition, Philadelphia, PA, United States, March 22-26, (2020), POLY-0388.

30. Smith, M. B.; March, J. Addition to Carbon-Hetero Multiple Bonds, In March's Advanced Organic Chemistry, J. Wiley \& Sons: New York, Ch.16, 1225 (2001). 